\title{
Predominance of ERG-negative high-grade prostate cancers in African American men
}

\author{
JAMES FARRELL ${ }^{1,2}$, DENISE YOUNG ${ }^{1}$, YONGMEI CHEN ${ }^{1}$, JENNIFER CULLEN ${ }^{1}$, INGER L. ROSNER ${ }^{1,2}$, \\ JACOB KAGAN $^{3}$, SUDHIR SRIVASTAVA ${ }^{3}$, DAVID G. MCLEOD ${ }^{1,2}$, ISABELL A. SESTERHENN ${ }^{4}$, \\ SHIV SRIVASTAVA ${ }^{1}$ and GYORGY PETROVICS ${ }^{1}$
}

\begin{abstract}
${ }^{1}$ Department of Surgery, Center for Prostate Disease Research, Uniformed Services University of the Health Sciences, Bethesda, MD 20814; ${ }^{2}$ Department of Urology, Walter Reed National Military Medical Center, Bethesda, MD 20889;

${ }^{3}$ Cancer Biomarkers Research Group, Division of Cancer Prevention, National Cancer Institute, National Institutes of Health, Bethesda, MD 20892; ${ }^{4}$ Department of Genitourinary Pathology, Joint Pathology Center, Silver Spring, MD 20910-1290, USA
\end{abstract}

Received May 26, 2014; Accepted July 29, 2014

DOI: $10.3892 / \mathrm{mco} .2014 .378$

\begin{abstract}
Erythroblast transformation-specific-related gene (ERG) fusions, the most common and validated prostate cancer $(\mathrm{CaP})$ genome alteration, result in alterations in the expression of the ERG oncoprotein. Significantly lower frequencies of ERG have been reported in tumors of African American (AA) in comparison to Caucasian American (CA) men. Building on our preliminary observations, this study has focused on the increased association of the ERG-negative status with higher-grade prostate tumors in AA men. Representative whole-mount prostate sections from a matched cohort of 63 AA and 63 CA men with Gleason scores of 4+3 and those with Gleason scores of 8-10 were analyzed for ERG oncoprotein by immunohistochemistry. The striking finding of this study was that ERG expression was 3 times more likely to be present in the higher-grade index tumors of CA men compared to AA men ( 31 of 63 vs. 10 of 63 patients, respectively; $\mathrm{P}<0.0001)$. Although the mechanisms underlying these differences have not been elucidated, the present study along with our previous observations underscores that ERG typing may enhance the understanding of ethnic differences and future targeted therapy of $\mathrm{CaP}$.
\end{abstract}

\section{Introduction}

African American (AA) men exhibit the highest incidence and mortality from prostate cancer $(\mathrm{CaP})$ compared to other

Correspondence to: Dr Shiv Srivastava or Dr Gyorgy Petrovics, Department of Surgery, Center for Prostate Disease Research, Uniformed Services University of the Health Sciences, 4301 Jones Bridge Road, Bethesda, MD 20814, USA

E-mail: ssrivastava@cpdr.org

E-mail: gpetrovics@cpdr.org

Key words: prostate cancer, erythroblast transformation-specificrelated gene, transmembrane protease serine 2 gene fusion, race, ethnicity races in the United States (1). While socioeconomic factors contribute to $\mathrm{CaP}$ outcomes among men of different ethnicities (2), it has also been recognized that AA men have more advanced $\mathrm{CaP}$ at diagnosis (3). Although there remains controversy over the role of biological differences between prostate tumors in AA and Caucasian American (CA) men, emerging data suggest the presence of differences in somatic and germline alterations $(4,5)$.

One of the most common and validated CaP genome alterations represents fusion of the protein-coding sequences of erythroblast transformation-specific (ETS)-related transcription factors [predominantly ETS-related gene $(E R G)]$ with promoter sequences of androgen-regulated genes [predominantly transmembrane protease serine 2 (TMPRSS2) gene] (6-9). The highly prevalent ERG fusions, present in over half of all CaPs in Western countries, result in androgen-dependent and prostate tumor-specific expression of the $E R G$ fusion transcripts and a near-full-length ERG protein with a 32-amino acid deletion at the amino terminus (6-9). Evaluations of the ERG alterations at the genomic, transcriptional and protein levels have continued to suggest lower frequencies of ERG in AA CaP in comparison to CA CaP (10-13). Almost complete concordance between the detection of $E R G$ gene fusions by fluorescence in situ hybridization and ERG protein detection by immunohistochemistry (IHC), has significantly accelerated the evaluation of the ERG protein as the surrogate of this common $\mathrm{CaP}$ genome alteration in pathological specimens (14-17). Studies from our and other groups indicate that the overall frequency of ERG alterations in CaP varies significantly among different ethnicities: It is highest in CA, intermediate in AA and lowest in Asian CaP patients $(4,5)$. Our recent evaluations of representative whole-mount prostate sections from a matched cohort of $91 \mathrm{CA}$ and 91 AA men demonstrated a significant difference $(\mathrm{P}<0.0001)$ in the prevalence of the ERG oncoprotein in index tumors of CA (63\%) and AA (29\%) men (13). Our preliminary data also suggested that the majority of higher-grade tumors in AA patients may be ERG-negative (13). The present study focuses on comparative evaluations of ERG in higher-grade tumors in CA and AA CaP patients. 
A

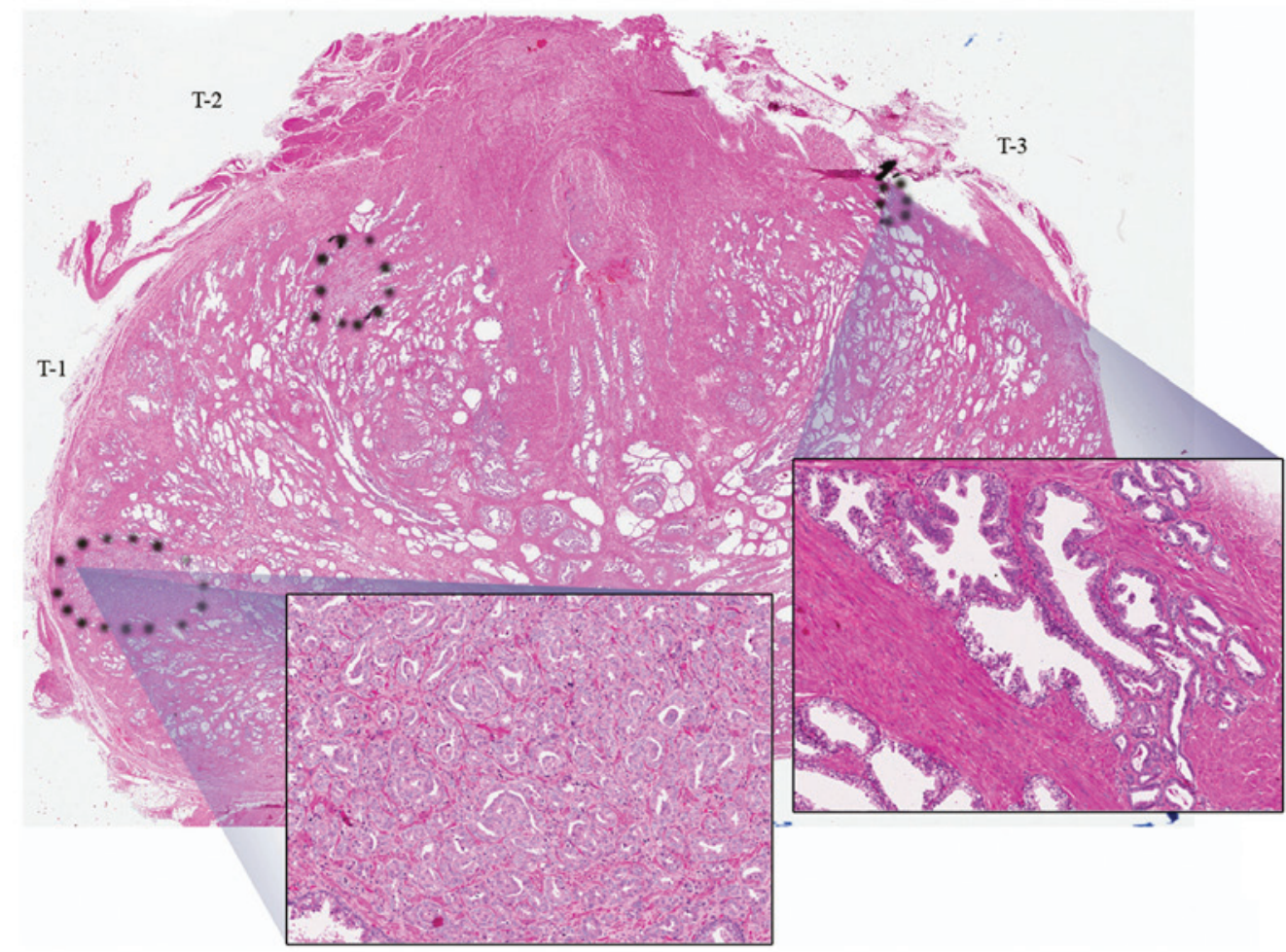

B

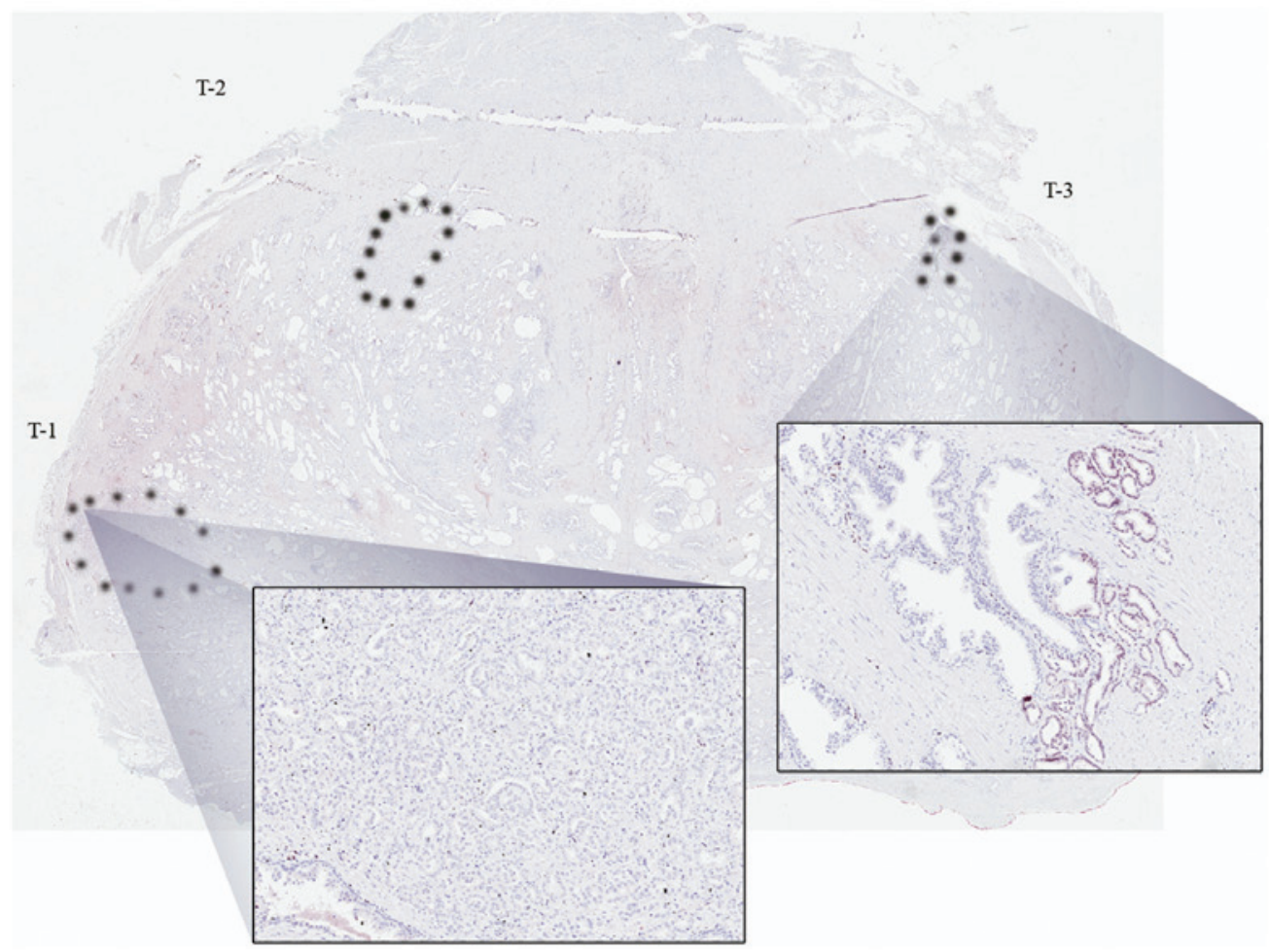

Figure 1. Representative images of whole-mount sections analyzed by hematoxylin and eosin (H\&E) staining, as well as ERG immunohistochemistry (IHC), with view fields enlarged (magnification, x20). (A) H\&E staining with tumor foci denoted by dotted outline. The higher power insert from T1 (index tumor) contains poorly differentiated (Gleason 4) disease. The T3 (tertiary tumor) insert on the right is well-differentiated (Gleason 3). (B) Analogous section with ERG IHC staining, in which the nuclear staining for ERG is negative in T1 and focally positive in T3.

\section{Materials and methods}

Specimens and study criteria. The Center for Prostate Disease Research database was queried to identify CaP patients who were enrolled in the Institutional Review Board-approved protocol from Walter Reed National Military Medical Center. The CaP patients underwent radical prostatectomy (RP) between 1994 and 2011. Archived clinicopathological data were evaluated for 1,304 patients who self-identified their race. The study sample was powered for ERG evaluation. A total of $63 \mathrm{AA}$ and $63 \mathrm{CA}$ 
Table I. Clinicopathological characteristics of all patients and breakdown across racial cohorts.

\begin{tabular}{|c|c|c|c|c|}
\hline Variables & All $(n=126)$ & AA $(n=63)$ & $\mathrm{CA}(\mathrm{n}=63)$ & P-value \\
\hline Age at RP, years & & & & 0.5887 \\
\hline Mean (SD) & $60.4(7.1)$ & $60.1(7.2)$ & $60.8(7.1)$ & \\
\hline PSA at diagnosis, ng/ml & & & & 0.2718 \\
\hline Median (range) & $6.7(0.9-5,065)$ & $6.9(1-5,065)$ & $6.5(0.9-23.4)$ & \\
\hline Pathological T stage & & & & 0.2008 \\
\hline pT2 & 49 (38.9) & $28(44.4)$ & $21(33.3)$ & \\
\hline pT3 or higher & $77(61.1)$ & $35(55.6)$ & $42(66.7)$ & \\
\hline Gleason sum & & & & 0.8538 \\
\hline $4+3$ & $47(37.3)$ & $24(38.1)$ & $23(36.5)$ & \\
\hline $8-10$ & 79 (62.7) & 39 (61.9) & $40(63.5)$ & \\
\hline ECE & & & & 0.6855 \\
\hline Negative & $49(43.0)$ & $26(44.8)$ & $23(41.1)$ & \\
\hline Positive & $65(57.0)$ & $32(55.2)$ & $33(58.9)$ & \\
\hline SV & & & & 0.2496 \\
\hline Negative & $91(72.8)$ & 48 (77.4) & $43(68.2)$ & \\
\hline Positive & $34(27.2)$ & $14(22.6)$ & $20(31.8)$ & \\
\hline Margin status & & & & 0.3230 \\
\hline Negative & $83(69.2)$ & $44(73.3)$ & $39(65.0)$ & \\
\hline Positive & $37(30.8)$ & 16 (26.7) & $21(35.0)$ & \\
\hline
\end{tabular}

AA, African American; CA, Caucasian American; RP, radical prostatectomy; SD, standard deviation; PSA, prostate-specific antigen; ECE, extracapsular extension; and SV, seminal vesicles invasion.

patients matched for age at RP and Gleason scores of 8-10 and $4+3$ of prostate tumors met the study inclusion criteria.

IHC analyses of the ERG. Representative whole-mount 4- $\mu \mathrm{m}$ cross-sections from each prostatectomy specimen were selected. The index tumor consisting of the largest tumor with the highest grade was identified along with all other tumor foci in each specimen. Specimens for ERG IHC were cut and stained with a highly specific anti-ERG monoclonal antibody (clone 9FY; Biocare Medical Inc., Concord, CA, USA) as previously described $(13,14)$. The index tumor and all other tumors were classified as ERG-positive (any number of tumor cells positive) or negative (all tumor cells negative). Fig. 1 provides representative examples.

Sample size and statistical analysis. Categorical patient clinicopathological data were described across race using frequencies and percentages. Continuously measured variables were compared using measures of central tendency, namely mean, median and standard deviation. The Chi-square test was used to compare the distribution of the clinicopathological characteristics between the CA and AA cohorts, as well as IHC status (positive vs. negative) for the AA vs. CA cohorts. Biochemical recurrence (BCR), was defined as 2 consecutive prostate-specific antigen (PSA) measurements of $\geq 0.2 \mathrm{ng} / \mathrm{ml}$ at least 8 weeks post-RP. Unadjusted Kaplan-Meier estimate curves and multivariable Cox proportion hazards analysis were used to evaluate the prognostic significance of ERG oncoprotein on BCR-free survival. The log-rank test was used to test for differences in the Kaplan-Meier curves by ERG status. $\mathrm{P}<0.05$ was considered to indicate a statistically significant difference. All data analyses were conducted using SAS software, version 9.3 (SAS Institute, Cary, NC, USA).

\section{Results}

Clinicopathological characteristics. The study cohort of 126 patients (63 CA and 63 AA) did not exhibit significant differences in clinicopathological variables across race (Table I). The majority of the tumors had Gleason scores of 8-10 and pT3 disease (Table I). This patient cohort provided an $80 \%$ power to detect a $25-30 \%$ absolute difference across race for ERG positivity (two-sided $\mathrm{P}$-value=0.05).

ERG status by race and grade. Overall, $46 \%$ of the patients had $\geq 1$ ERG-positive tumor foci. The index tumor was ERG-positive in 41 of the 126 patients. In CA men, the index tumor was ERG-positive in 31 of 63 patients (49\%), which was significantly higher compared to 10 of 63 patients $(16 \%)$ in AA men $(\mathrm{P}<0.0001)$ (Table II). CA men were also significantly more likely to have any tumor focus positive for ERG compared to AA men (59 vs. $41 \%, \mathrm{P}=0.0042$, data not shown). ERG-positive status was significantly lower in higher-grade (16\%) compared to lower-grade (34\%) index tumors of AA men $(\mathrm{P}=0.04)$, which was not the case in CA men (Table II).

ERG as a predictor of recurrence. ERG was not found to be an independent predictor of BCR in this cohort (Table III). 
Table II. Prevalence of ERG positivity across race in high-grade (Gleason score, 8-10 and 4+3) index tumors (upper lane, present study) and in low-grade (Gleason score, 6) index tumors (lower lane).

\begin{tabular}{lccc}
\hline ERG status/grade & Total & CA & AA \\
\hline ERG+/high-grade & $33 \%(41 / 126)$ & $49 \%(31 / 63)$ & $16 \%(10 / 63)$ \\
ERG+/low-grade & $52 \%(35 / 67)$ & $69 \%(24 / 35)$ & $34 \%(11 / 32)$ \\
P-value & & 0.0642 & 0.0400 \\
\hline
\end{tabular}

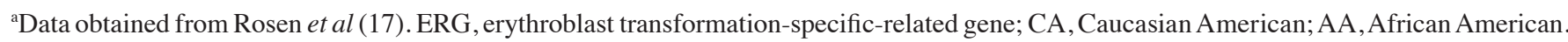

Table III. Univariable and multivariable Cox proportional hazard models for the prediction of biochemical recurrence by using ERG IHC status and clinicopathological variables.

\begin{tabular}{|c|c|c|c|c|}
\hline \multirow[b]{2}{*}{ Variables } & \multicolumn{2}{|c|}{ Univariable Cox models } & \multicolumn{2}{|c|}{ Multivariable Cox model } \\
\hline & $\mathrm{HR}(95 \% \mathrm{CI})$ & P-value & $\mathrm{HR}(95 \% \mathrm{CI})$ & P-value \\
\hline Age at RP & $1.011(0.965-1.059)$ & 0.6481 & & \\
\hline Log PSA & $1.352(1.062-1.723)$ & 0.0145 & $1.289(0.993-1.674)$ & 0.0564 \\
\hline \multicolumn{5}{|l|}{ Race/ethnicity } \\
\hline $\mathrm{CA}$ & 1 & & & \\
\hline $\mathrm{AA}$ & $0.705(0.371-1.340)$ & 0.2866 & & \\
\hline \multicolumn{5}{|c|}{ Pathological T stage } \\
\hline pT2 & 1 & & 1 & \\
\hline pT3 or higher & $4.737(1.972-11.379)$ & 0.0005 & $5.749(1.729-19.115)$ & 0.0043 \\
\hline \multicolumn{5}{|l|}{ Gleason sum } \\
\hline $4+3$ & 1 & & 1 & \\
\hline $8-10$ & $1.858(0.879-3.928)$ & 0.1048 & $1.272(0.545-2.968)$ & 0.5777 \\
\hline \multicolumn{5}{|l|}{ SV } \\
\hline Negative & 1 & & 1 & \\
\hline Positive & $2.240(1.183-4.241)$ & 0.0133 & $1.159(0.571-2.354)$ & 0.6827 \\
\hline \multicolumn{5}{|l|}{ Margin status } \\
\hline Negative & 1 & & 1 & \\
\hline Positive & $2.276(1.193-4.342)$ & 0.0126 & $0.890(0.427-1.855)$ & 0.7562 \\
\hline \multicolumn{5}{|l|}{ ERG IHC status } \\
\hline ERG- & 1 & & & \\
\hline ERG+ & $1.366(0.704-2.652)$ & 0.3564 & & \\
\hline
\end{tabular}

ERG, erythroblast transformation-specific-related gene; IHC, immunohistochemistry HR, hazard ratio; CI, confidence interval; RP, radical prostatectomy; PSA, prostate-specific antigen; CA, Caucasian American; AA, African American; SV, seminal vesicles. P-values in bold print denote statistically significant differences $(<0,05)$.

Pathological stage was an independent predictor of BCR [hazard ratio $(\mathrm{HR})=5.749, \mathrm{P}=0.0043$ ] and there was a trend towards higher serum PSA levels at diagnosis $(\mathrm{HR}=1.289$, $\mathrm{P}=0.0564$ ) (Table III).

\section{Discussion}

$\mathrm{CaP}$ is a multifocal, heterogeneous disease with a variable clinical course. Two cancers of the same grade and stage do not necessarily exhibit similar progression characteristics and $\mathrm{CaP}$ does not behave equally across age groups or ethnicities (1-5,18). Molecular alterations are likely involved in the ethnic differences of $\mathrm{CaP}$ and we sought to describe the prevalence of ERG in higher-grade disease in AA and CA men with a focus on index tumors. High Gleason scores are recognized as surrogates of aggressive disease and are independently predictive of BCR (19).

Studies from our and other groups have demonstrated significantly lower frequencies of ERG in $\mathrm{CaP}$ of AA men in comparison to that of CA men $(5,12,13)$. Our previous preliminary observation indicated more significant differences in ERG in high-grade tumors of AA compared to those of CA men. 
This adequately powered study addressed this issue by using matched cohorts of $\mathrm{CA}$ and $\mathrm{AA} \mathrm{CaP}$ specimens. A striking finding of this study was that ERG was significantly (3 times) more likely to be present in the higher-grade index tumors of CA men compared to those of AA men (31 of 63 vs. 10 of 63 patients, respectively; $\mathrm{P}<0.0001)$. Thus, although ERG may be the most common oncogenic alteration in CA men, it does not appear to be the case in AA men, particularly not in those with higher-grade $\mathrm{CaP}$. The biological basis underlying this observation remains to be elucidated; these results nonetheless support the association of an ERG-negative status with more aggressive disease in AA men. These data also suggest that ERG may not be the primary driver of higher-grade $\mathrm{CaP}$ in AA men.

While there is a general agreement that $E R G$ is a highly prevalent and early oncogenic alteration in $\mathrm{CaP}$ and it defines a large subtype of prostate tumors, it is also important to recognize that there are significant proportions of ERG-negative prostate tumors for which a common driver gene alteration is not known. Emerging data from the present and other studies underscore the higher prevalence of the ERG-negative subtype of $\mathrm{CaP}$ in $\mathrm{AA}$ and Asian men $(4,5)$. The higher frequency of high-grade ERG-negative tumors in AA men likely reflects the presence of distinct genomic alterations associated with the initiation and progression of this subtype of $\mathrm{CaP}$.

The utility of ERG detection in $\mathrm{CaP}$ is apparent in the diagnostic setting and ERG typing of tumors may also be of significant value for biological classification and future targeted therapy. However, the utility of ERG in assessing $\mathrm{CaP}$ progression remains controversial, which may be attributed to multifactorial causes, including specific patient cohort, disease stage and assay type $(8,17)$. In this high-grade cohort, the ERG protein status was not found to be correlated with disease progression.

In summary, this study provides important observations on the predominance of ERG-negative high-grade $\mathrm{CaP}$ in AA men. The biological implications of these observations are far-reaching, particularly in delineating biological typing and future treatment of $\mathrm{CaP}$ tumors in men of different ethnicities.

\section{Acknowledgements}

The views expressed in this review do not reflect any official policy of the Department of the Army, Department of Defense, or the US Government. We wish to thank Mr. Stephen Doyle for the artwork.

\section{References}

1. Siegel R, Naishadham D and Jemal A: Cancer statistics, 2013. CA Cancer J Clin 63: 11-30, 2013.

2. Schwartz K, Powell IJ, Underwood W III, et al: Interplay of race, socioeconomic status and treatment on survival of patients with prostate cancer. Urology 74: 1296-1302, 2009.

3. Williams H and Powell IJ: Epidemiology, pathology and genetics of prostate cancer among African Americans compared with other ethnicities. Methods Mol Biol 472: 439-453, 2009.

4. Martin DN, Starks AM and Ambs S: Biological determinants of health disparities in prostate cancer. Curr Opin Oncol 25: 235-241, 2013.

5. Farrell J, Petrovics G, McLeod DG and Srivastava S: Genetic and molecular differences in prostate carcinogenesis between African American and Caucasian American men. Int J Mol Sci 14: 15510-15531, 2013.

6. Tomlins SA, Rhodes DR, Perner S, et al: Recurrent fusion of TMPRSS2 and ETS transcription factor genes in prostate cancer. Science 310: 644-648, 2005.

7. Petrovics G, Liu A, Shaheduzzaman S, et al: Frequent overexpression of ETS-related gene-1 (ERG1) in prostate cancer transcriptome. Oncogene 24: 3847-3852, 2005.

8. Rubin MA, Maher CA and Chinnaiyan AM: Common gene rearrangements in prostate cancer. J Clin Oncol 29: 3659-3668, 2011.

9. Barbieri CE, Bangma $\mathrm{CH}$, Bjartell A, et al: The mutational landscape of prostate cancer. Eur Urol 64: 567-576, 2013.

10. Hu Y, Dobi A, Sreenath T, et al: Delineation of TMPRSS2-ERG splice variants in prostate cancer. Clin Cancer Res 14: 4719-4725, 2008.

11. Rice KR, Chen Y, Ali A, et al: Evaluation of the ETS-related gene mRNA in urine for the detection of prostate cancer. Clin Cancer Res 16: 1572-1576, 2010.

12. Magi-Galluzzi C, Tsusuki T, Elson P, et al: TMPRSS2-ERG gene fusion prevalence and class are significantly different in prostate cancer of Caucasian, African-American and Japanese patients. Prostate 71: 489-497, 2011.

13. Rosen P, Pfister D, Young D, et al: Differences in frequency of ERG oncoprotein expression between index tumors of Caucasian and African American patients with prostate cancer. Urology 80: 749-753, 2012.

14. Furusato B, Tan SH, Young D, et al: ERG oncoprotein expression in prostate cancer: clonal progression of ERG-positive tumor cells and potential for ERG-based stratification. Prostate Cancer Prostatic Dis 13: 228-237, 2010.

15. Park K, Tomlins SA, Mudaliar KM, et al: Antibody-based detection of ERG rearrangement-positive prostate cancer. Neoplasia 12: 590-598, 2010.

16. Braun M, Goltz D, Shaikhibrahim Z, et al: ERG protein expression and genomic rearrangement status in primary and metastatic prostate cancer - a comparative study of two monoclonal antibodies. Prostate Cancer Prostatic Dis 15: 165-169, 2012.

17. Rosen P, Sesterhenn IA, Brassell SA, et al: Clinical potential of the ERG oncoprotein in prostate cancer. Nat Rev Urol 9: 131-137, 2012.

18. Brassell SA, Rice KR, Parker PM, et al: Prostate cancer in men 70 years old or older, indolent or aggressive: clinicopathological analysis and outcomes. J Urol 185: 132-137, 2011.

19. Brimo F, Montironi R, Egevad L, et al: Contemporary grading for prostate cancer: implications for patient care. Eur Urol 63: 892-901, 2013. 